\title{
NOMINAL LONGITUDINAL PARAMETERS FOR THE LHC BEAM IN THE CERN SPS
}

\author{
P. Baudrenghien, T. Bohl, T. Linnecar, E. Shaposhnikova and J. Tuckmantel, \\ CERN, Geneva, Switzerland
}

\section{Abstract}

A proton beam with the basic structure defined by the the LHC requirements, was first available for injection into the SPS in 1998. At the end of 2002, following a significant beam-studies and RF hardware upgrade programme, a beam having both the nominal LHC intensity and the correct longitudinal parameters was obtained at top energy for the first time. This beam, characterised by high local density, must satisfy strict requirements on bunch length, longitudinal emittance and bunch to bunch phase modulation for extraction to the LHC, where only very limited particle losses are acceptable. The problems to be solved came mainly from the high beam loading and microwave and coupled bunch instabilities which led both to beam losses and to unacceptably large longitudinal emittance on the flat top. In this paper the steps taken to arrive at these nominal beam parameters are presented.

\section{INTRODUCTION}

The preparation of the CERN SPS for its future role as an injector of LHC started well before the first LHC type beam was available from the PS injector. At the beginning work concentrated on single bunch stability. During the studies of microwave instability, which started in 1995, the guilty impedances were identified and then the sources shielded two years ago. Single bunch reference measurements before and after the impedance reduction are summarised below together with final results for the whole LHC beam. The nominal LHC beam consists of 3 or 4 batches with 72 bunches each. Bunches are spaced by $25 \mathrm{~ns}$ and batches by $220 \mathrm{~ns}$. Nominal bunch intensity at top energy is $1.1 \times 10^{11}$.

To cope with expected large longitudinal emittances, it was suggested in 1998 to install an additional $200 \mathrm{MHz}$ RF system in the LHC for beam capture and injection damping. In this case bunches with an emittance $\varepsilon$ around $1 \mathrm{eVs}$ would be acceptable. For loss-free capture in the LHC with the main $400 \mathrm{MHz}$ RF system alone, in the presence of expected energy and phase errors, $\varepsilon$ should be less than $0.7 \mathrm{eVs}$ and the bunch length $\tau<2$ ns. At injection into the SPS $(26 \mathrm{GeV}) \varepsilon=0.35 \mathrm{eVs}$.

The LHC beam was first injected with bunch and total intensity much lower than nominal. Even so it was unstable first on the flat bottom, then during the ramp after $5 \mathrm{~s}$ of acceleration $(280 \mathrm{GeV})$, and again on the flat top $(450 \mathrm{GeV})$.

The continuous emittance blow-up due to the microwave instability, leading to beam losses on the flat bottom, had disappeared in 2001, after the impedance reduction. From then on the main efforts to obtain nominal LHC beam concentrated on commissioning new hardware to cope with strong beam loading and instabilities caused by the impedance of the main $200 \mathrm{MHz}$ RF system, and on cures for the coupled bunch instabilities observed at the end of the acceleration ramp.

\section{IMPEDANCE REDUCTION}

The impedance reduction programme in the SPS was completed during the shutdown of 2000/2001. Around 1000 vacuum ports were shielded [1], different types of kickers and septa were screened, and all lepton equipment including $3 \mathrm{RF}$ systems was removed from the ring.

The single bunch reference measurements in 2001 have demonstrated significantly improved stability [2]. The change of quadrupole synchrotron frequency shift with intensity by a factor 2.5 agrees well with that expected from the reduction in the low frequency inductive impedance estimated from the impedance budget (from $12.4 \mathrm{Ohm}$ to $5.6 \mathrm{Ohm})$. The space charge impedance at $26 \mathrm{GeV}$ is $-1 \mathrm{Ohm}$. The slope of the bunch lengthening curve is reduced by a factor 7; emittance blow-up due to the microwave instability is seen before the impedance reduction but bunch lengthening due to the potential well distortion after. In the measurements of bunch spectra with RF off, the resonant peaks due to the vacuum ports (above $1.3 \mathrm{GHz}$ ) and kickers (at $400 \mathrm{MHz}$ ) have disappeared.

For the LHC type beam, no emittance blow-up is observed now on the flat bottom, and even some controlled emittance blow-up is necessary as a cure for coupled bunch instabilities at high energies (see below).

The impedance of the main RF system around the fundamental $(200 \mathrm{MHz}$ ) frequency had long been recognized to be a serious problem not only for beam loading but also for coupled-bunch instability [3]. The One-Turn-Delay Feedback (1tFB) in operation since the early eighties [4] and acting on the 4 cavities in parallel did not provide sufficient impedance reduction for the LHC beam. By April 2001 all cavities had been equipped with a Feedforward system providing a (10-15) $\mathrm{dB}$ impedance reduction in a $1 \mathrm{MHz}$ band on each side of the RF frequency [5]. By the end of 2001 an upgraded $1 \mathrm{tFB}$ had also been installed on each cavity ( $20 \mathrm{~dB}$ impedance reduction at the RF frequency, $1 \mathrm{MHz}$ single-sided bandwidth). However a coupled-bunch instability (dipole mode) with frequency $<2 \mathrm{MHz}$ was still observed on the flat bottom at half nominal intensity. In 2002 the feedback bandwidth was therefore increased $(2 \mathrm{MHz}$ single-sided) and two cavities were equipped with a longitudinal damping system capable of damping dipole modes up to $3 \mathrm{MHz}$ during the full acceleration ramp.

In 2003 three of the $200 \mathrm{MHz}$ cavities will have new 
power couplers. They are rated for more than $1 \mathrm{MW}$ continuous power per cavity. With improved multipactoring control they should also allow low voltage without counterphasing, thus easing operation under beam loading [7].

In 2002, after the scrubbing run at the beginning of the operation year, the nominal intensity could be reached without serious problems from vacuum pressure increase due to e-cloud [6]. As a result of all upgrades to the $200 \mathrm{MHz}$ RF system, this beam was stable on the flat bottom. However a coupled bunch instability was observed at $280 \mathrm{GeV}(16 \mathrm{~s})$ for a single batch with an intensity as low as $2 \times 10^{12}$. The source of this instability is not yet clear; one of the high order modes (with mode frequency outside the bandwidth of the longitudinal damper) in the $200 \mathrm{MHz}$ cavities is suspected.

\section{INSTABILITY AT HIGH ENERGY}

\section{Effect of voltage programme}

The main purpose of these studies was to optimise the $200 \mathrm{MHz}$ voltage $\left(V_{1}\right)$ through the cycle to obtain minimum emittance and bunch length on the flat top. As a basis we used a voltage programme for $\varepsilon=0.5 \mathrm{eVs}$ with a constant filling factor in momentum of 0.95 .
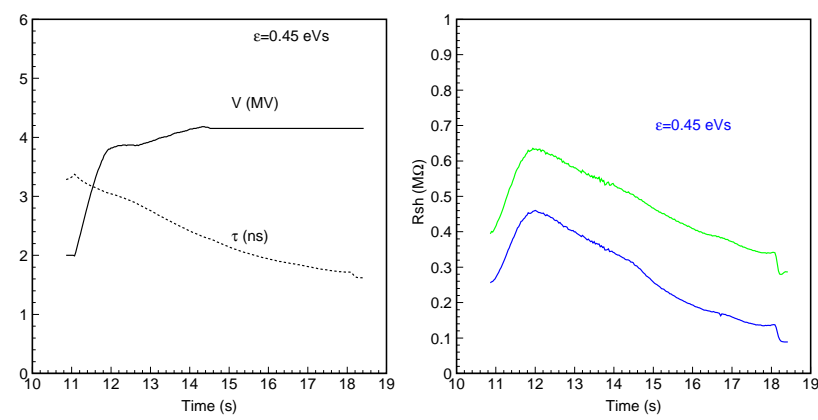

Figure 1: Left: the $200 \mathrm{MHz}$ voltage programme used during studies together with the expected variation of bunch length for $\varepsilon=0.45 \mathrm{eVs}$. Right: the minimum threshold impedance for coupled bunch instabilities for this voltage programme with $800 \mathrm{MHz}$ off (bottom curve) and on (top).

In all the cases studied the beam was unstable well below the nominal intensity. The emittance measured on the flat top was larger for higher voltage during the cycle, and increased also with intensity. For the voltage programme in Fig. 1 (left), $\varepsilon=0.73$ eVs was obtained at $450 \mathrm{GeV}$ for a single batch with nominal intensity at injection $(\sim 10 \%$ lower at $450 \mathrm{GeV}$ ). Measurements of bunch length (averaged over the last 5 bunches in the batch, which are the most unstable ones and can have up to $50 \%$ more emittance blow-up than the first bunches) through this cycle are presented in Fig. 2 (top left) and should be compared with the expected behaviour during the cycle from Fig. 1 (left).

Measurements in 2000 for 48 bunches with intensity $8.3 \times 10^{10} /$ bunch $\left(V_{1}=3.5 \mathrm{MV}\right.$ at the end of the ramp),
Time $[1000 * \mathrm{~ms}]$

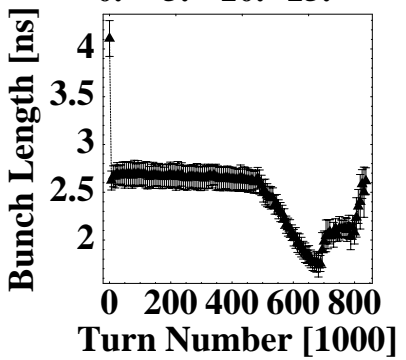

Time $[1000 * \mathbf{m s}]$

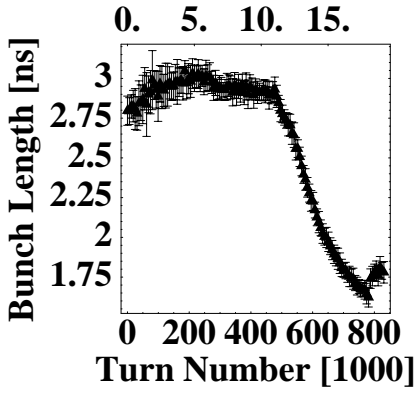

0. 5. 10. 15.
0. 5. 10. 15.

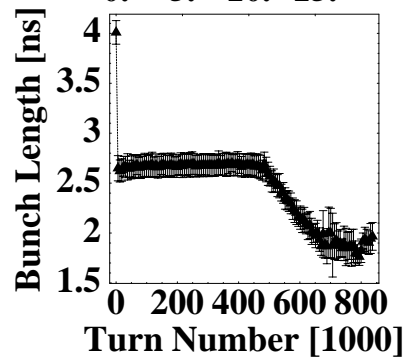

Time $[1000 * \mathbf{m s}]$

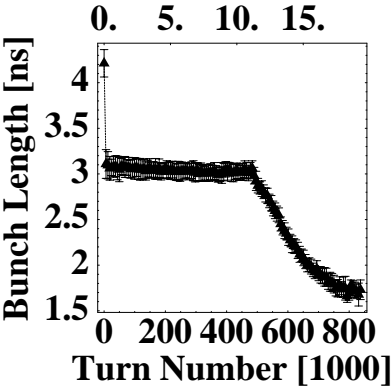

Time $[1000 * \mathrm{~ms}]$

Figure 2: The average bunch length during the cycle with $V_{1}$ from Fig. 1 and $800 \mathrm{MHz}$ off (top left), $800 \mathrm{MHz}$ on during the ramp (top right), $800 \mathrm{MHz}$ on from injection (bottom left) and $800 \mathrm{MHz}$ on during the ramp plus emittance blow-up due to mismatched injection (bottom right). Single batch with injected intensity $7.5 \times 10^{12}$.

gave $\varepsilon=1.1 \mathrm{eVs}$ [8]. This should be compared with $0.53 \mathrm{eVs}$ obtained under similar conditions in 2002.

On the flat bottom an increase of voltage improves the beam stability. This is why the matched voltage of $0.75 \mathrm{MV}$ used for beam capture was adiabatically raised to $2 \mathrm{MV}$, this being repeated 4 times (for each injection). Increasing the voltage up to $7 \mathrm{MV}$ on the flat top, necessary to shorten the bunches before transfer to LHC, has the opposite effect. The beam is more stable at lower voltage. This can be explained by the fact that at $26 \mathrm{GeV}$ the instability is due to the $200 \mathrm{MHz}$ impedance, while at the top energy it is due to a higher frequency impedance, so that reducing the bunch length has the opposite effect [9]. The decrease of the threshold towards the end of the cycle and the sharp drop on the flat top in Fig. 1 explain very well the instability observed at $16 \mathrm{~s}$ and then later again on the flat top.

\section{High harmonic RF system}

The stabilising effect of the $800 \mathrm{MHz}$ RF system can be seen in Fig. 1 (right) and Fig. 2 (top right). The total voltage seen by the beam in the presence of two RF systems is:

$$
V=V_{1} \sin \phi+V_{2} \sin (4 \phi+\Delta \phi) .
$$

The phase shift $\Delta \phi$ was programmed through the cycle to increase the synchrotron frequency spread using the bunch 
shortening (BS) mode: $\Delta \phi=\pi-4 \phi_{s}$. The $800 \mathrm{MHz}$ voltage was limited through the cycle to the level $V_{2} / V_{1} \simeq 0.1$ [10] to avoid creation of a flat zone in the synchrotron frequency distribution outside the bunch center, where Landau damping can be lost.

Until the effect of beam loading in the idling $800 \mathrm{MHz}$ cavity was taken into account, there was a large difference in the phase shift $\Delta \phi$ required for BS mode between the flat bottom and flat top due to the beam induced voltage. Correction of this phase shift eliminated additional emittance blow-up, Fig. 2 (bottom left). As a result the beam became unstable on the flat top and a small controlled emittance blow-up had to be introduced.

\section{Controlled emittance blow-up}

The optimum (minimum) controlled emittance blow-up in the SPS should ensure at the same time both beam stability and minimum loss at the SPS-LHC bunch to bucket transfer. A first blow-up on the flat bottom by mismatched injection ( $V=2 \mathrm{MV}$ instead of $0.75 \mathrm{MV}$ ) gives an increase of emittance from $0.35 \mathrm{eVs}$ to $0.4 \mathrm{eVs}$, Fig. 2 (bottom right). This was sufficient to stabilise the beam with injected intensities up to nominal. To obtain nominal intensities with $7 \mathrm{MV}$ on the flat top an additional emittance blow-up was needed, see Fig. 3.
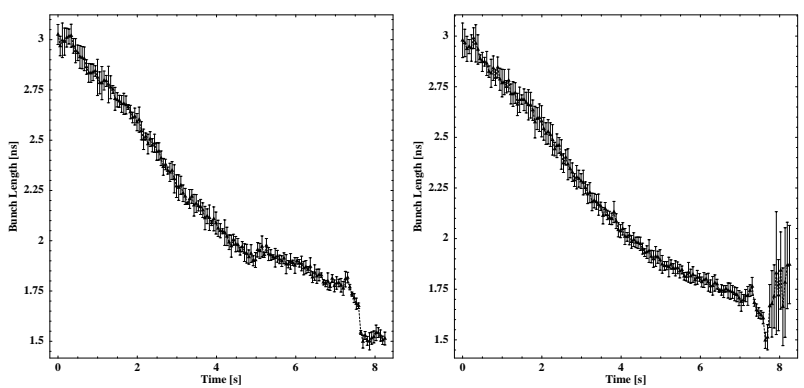

Figure 3: Effect of controlled emittance blow-up on beam stability. The average (of the 5 last bunches in the batch) bunch length during the ramp with bunch excitation (left) and without (right). $1.07 \times 10^{11} /$ bunch at $450 \mathrm{GeV}$.

Two methods were used for emittance blow-up during acceleration. First, by phase modulation of the $800 \mathrm{MHz}$ voltage, see (1),

$$
\Delta \phi(t)=\alpha \sin \left(2 \pi f_{\text {mod }}+\psi_{0}\right)+\Delta \phi_{0}
$$

The resonant excitation at $16 \mathrm{~s}$ during $10 \mathrm{~ms}$ (bucket area $0.75 \mathrm{eVs}$ ) provided an emittance increase to $0.6 \mathrm{eVs}$. This was done using parameters optimised for the 4th harmonic [11] $f_{\text {mod }}=3 f_{s}, \alpha=1.05$ and $\Delta \phi_{0}=\pi$, where $f_{s}$ is the linear synchrotron frequency.

Second, pink noise excitation of the $200 \mathrm{MHz}$ amplitude was also tried. It was applied with the $800 \mathrm{MHz}$ in BS mode (to affect the bunch center more than the tails) at $15.8 \mathrm{~s}$ at frequency $2 f_{s}$ with bandwidth $\pm \Delta f / f_{s} \simeq 0.125$.
It was less effective (a blow-up to $0.54 \mathrm{eVs}$ after $0.5 \mathrm{~s}$ ) but could be useful for LHC, where a high harmonic RF system will not be available. Small beam losses at the time of excitation were observed for both methods. Decreasing the time or strength of excitation eliminated losses but then the emittance blow-up was insufficient to stabilise beam on the flat top. A possibly better technique using a programmable excitation frequency is planned for 2003 .

\section{SUMMARY}

Bunch lengths ( $4 \sigma$ Gaussian fit) in the range (1.51 1.58) ns, depending on the batch number, and averaged over 50 bunches, were finally obtained on the flat top for 4 batches in the ring with the nominal bunch intensity $1.1 \times 10^{11}$. These bunch lengths correspond to emittances of $(0.52-0.56) \mathrm{eVs}$, well below our initial target. These bunch parameters together with the bunch to bunch phase modulation of $\pm 0.065 \mathrm{~ns}$ achieved $\left(V_{1}=7 \mathrm{MV}\right)$, the expected energy error $\pm 50 \mathrm{MeV}$ and reserve for an eventual synchronisation error (say $\pm 0.13 \mathrm{~ns}$ ) should provide minimum particle loss at injection into the LHC even in the absence of the $200 \mathrm{MHz}$ RF system, which consequently will not be installed in the LHC at least for nominal intensities.

These results have been achieved due to the SPS impedance reduction, the commissioning of improved feedback, feedforward and damping systems based on the $200 \mathrm{MHz}$ RF system and the use of the 4-th harmonic RF system both for beam stabilisation by increasing synchrotron frequency spread through the cycle and controlled emittance blow-up during the ramp.

We are grateful to the operation teams for their help, to G. Lambert, R. Olsen, D. Stellfeld and U. Wehrle for their assistance and the considerable electronics development and to E. Montesinos for excellent collaboration.

\section{REFERENCES}

[1] P. Collier et al., Proceed. of EPAC 2002, Paris, p. 1458.

[2] T. Bohl, T. Linnecar, E. Shaposhnikova, Proceed. of EPAC 2002, Paris, p. 1446.

[3] D. Boussard, G. Dome, T.P.R. Linnecar, IEEE Transactions on Nuclear Science, Vol. NS-26, No. 3, June 1979.

[4] D. Boussard, G. Lambert, IEEE Transactions on Nuclear Science, Vol. NS-30, No. 4, August 1983

[5] P. Baudrenghien, G. Lambert, Proc. LHC Workshop Chamonix XI, CERN-SL-2001-003 DI, p. 63, 2001

[6] G. Arduini et al., The LHC Proton Beam in the CERN SPS, these Proceed.

[7] T. Bohl, Proc. Workshop Chamonix X, CERN-SL-2000-007 DI, p. 56, 2000.

[8] T. Bohl, Proc. LHC Workshop Chamonix XI, CERN-SL2001-003 DI, p. 73, 2001.

[9] E. Shaposhnikova, CERN SL-Note-2001-031 HRF, 2001.

[10] T. Bohl, T. Linnecar, E. Shaposhnikova, J. Tuckmantel, Proceed. EPAC 1998, Stockholm, p. 978.

[11] T. Bohl et al., CERN SL-MD Note 221, 1996. 